\title{
CIRCUIT DESIGN BASED ON CAPACITIVE MICROMECHANICAL GYROSCOPE
}

\author{
Honglei Shen \\ Suzhou Industrial Park Industrial Technology School, \\ Jiangsu Lianhe Technical Institute, Jiangsu, China \\ E-mail: shenhongleishl@163.com
}

\begin{abstract}
The purpose is to study the circuit design based on capacitive micromechanical gyroscope. In order to detect a very weak angular rate signal, the signal needs to be properly amplified and demodulated. To this end, an improved continuous-time front-end readout circuit is proposed. The front-end structure is a charge-sensitive amplifier (CSA) with a T-resistor network as a feedback resistor. In order to reduce the noise of the front-end amplifier, the size of the input transistor is optimized accordingly. In addition, in the demodulation process, the comparator demodulation clock signal usually has a certain phase shift. Therefore, this will cause errors in the output signal. For this problem, a simple and effective error correction algorithm is put forward. By the validation of simulation, the results show that the error rate is reduced from $13.7 \%$ to $0.3 \%$ after the error correction algorithm. Therefore, it can be concluded that the circuit can correctly demodulate the angular rate signal. The algorithm can effectively reduce the error caused by demodulation.
\end{abstract}

Keywords: Micro-Mechanical Gyroscope, Readout Circuit, Front-End Amplifier, Demodulation.

\section{Introduction}

The gyroscope is a sensor for sensing the angle or angular motion of an object. It is the core components of various inertial control systems. Combined with the accelerometer, the gyroscope can track and capture the complete motion of the object in three-dimensional space, and realize accurate navigation, positioning and attitude control [3]. With the development of semiconductor manufacturing and microelectronics, physical structures such as sensors and actuators are integrated with microelectronic circuits on the same substrate. Thus,

A micro-electro-mechanical system (MEMS) is formed [1]. Compared with traditional gyroscopes, micromechanical gyroscope has great advantages. It includes small integration, low power consumption, low cost, long life, high reliability and good dynamic performance [2].

In the micromechanical gyro system, the readout circuit plays an important role. Its function is to complete the conversion from the micro-mechanical physical signal to the electrical signal, and from which to obtain an electrical signal proportional to the input angular rate. Because micromachined gyroscope adopts micromachining technology, it makes sensor signal very weak [4]. For capacitive micromechanical gyroscopes, the signal is a capacitance change signal. At the excitation of a typical angular rate signal, the amount of capacitance change is on the order of aF (10 -18F). Thus, the readout circuit is required to have characteristics such as low noise and high sensitivity. The noise and sensitivity of the readout circuit depends primarily on the front-end capacitance detection circuit and the angular rate demodulation. Therefore, the research and design of the front-end capacitance detection circuit and the angular rate demodulation are of great significance to improve the performance of the readout circuit and the performance of the whole micro-mechanical gyroscope.

\section{State of the art}

Micro-mechanical gyroscope has a wide range of applications. In the 80 s of the last century, a great deal of research was done abroad. However, the early development of micro mechanical gyroscope technology has a high risk, which led to private companies do not want to take risks alone.

Therefore, the research funding of MEMS gyroscope mostly comes from the government abroad. The United States relies mainly on universities [5]. The main MEM research university is Stanford University, Georgia Institute of Technology, University of California, Los Angeles, and Dreiberi Laboratory (CSDL). Europe has a national research in France LETI (Information and Electronic Technology Laboratory). Germany has a non-profit Fraunhofer Institute and the German 
Institute for Microtechnology. In addition, there are some universities and research institutes in Switzerland. In 2002, ADI published a gyro system program. The gyro adopts the capacitive micromechanical gyroscope of the body processing method. The micromachined structure and the readout circuit are integrated on the same substrate [6]. It is manufactured by using $3 \mathrm{umBiCOMS}$ process, and the noise is $50 /(\mathrm{h} \cdot \mathrm{Hz})$. The drive loop consists of a Transimpedance Amplifier (TIA) and a comparator, but this loop has no amplitude control circuitry. Finland Helsinki University of Technology published its micro-gyroscope system. Both the drive loop and the readout circuit use a chargesensitive amplifier (CSA) as the front-end C / V conversion circuit. The signal is filtered, amplitude adjusted, and so on. The two-band sigma-delta modulator digitizes the signal. The DSP module implemented by FPGA can perform data extraction, filtering, driving mode oscillation, amplitude stabilization, angular rate signal demodulation and so on [7].

To sum up, the existing micromachined gyroscopes still have many defects. In order to reduce the noise of the front-end amplifier, the size of the input transistor is optimized accordingly. In addition, in the demodulation process, the comparator demodulation clock signal usually has a certain phase shift.
Therefore, this will cause errors in the output signal. For this problem, a simple and effective error correction algorithm is put forward.

By the validation of simulation, the results show that the algorithm can effectively reduce the error rate of the output angular rate signal.

\section{Methodology}

\subsection{Electrical model and parameters of micro - mechanical gyroscope}

Figure 1 is a simple capacitive model of capacitive micromachined gyroscope. Since the drive is not used to detect the capacitance of the gyro, it is omitted in the figure for the sake of brevity.

Among them, dr+and dr- denotes the driving electrodes, sen + and sen- are the detecting electrodes. The $\mathrm{Cd} 1$ and $\mathrm{Cd} 2$ are driving capacitors, and the Cs1 and Cs2 are the detection capacitors. The Cds2 is the coupling capacitor that drives the lower plate and detects the lower plate. The Cpstis the parasitic capacitance of the upper plate, and the Cpsbis the parasitic capacitance of the lower plate. The Cpcis the parasitic capacitance of the intermediate plate.

Table 1 gives descriptions and values for other important parameters.

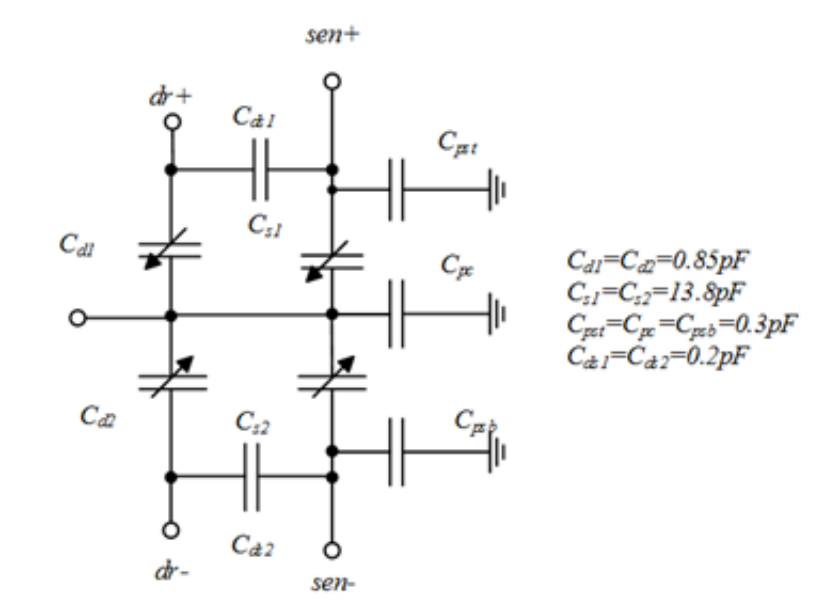

Figure 1: The capacitance model of capacitive micromachined gyroscope

Table 1. The description and numerical values of other parameters of the gyro

\begin{tabular}{|c|c|c|}
\hline Symbol & Description & Value \\
\hline $\mathrm{fx}$ & Drive modal resonant frequency & $4.2 \mathrm{kHz}$ \\
\hline $\mathrm{Qx}$ & Drive modal quality factor & 50000 \\
\hline $\mathrm{fy}$ & Detection of modal resonant frequency & $4.7 \mathrm{kHz}$ \\
\hline $\mathrm{Qy}$ & Detection of modal quality factor & 20000 \\
\hline$\Delta \mathrm{Csr}$ & Maximum differential range of detection capacitor & $100 \mathrm{fF}$ \\
\hline
\end{tabular}




\subsection{Readout circuit structure}

The maximum detection capacitance of gyroscope is $\mathrm{s}$, maxC is $100 \mathrm{fF}$, and corresponding input angle rate is $726^{\circ} / \mathrm{s}$. The angular rate input bandwidth is $100 \mathrm{~Hz}$.

According to the research in the previous two chapters, the front-end detection circuit uses the non-modulation continuous time voltage detection, to realize the conversion from capacitive signal to voltage signal, that is to say, $\mathrm{C} / \mathrm{V}$ conversion. The demodulator of the coupling switch is used for demodulation of the angular rate signal and the angular rate signal is removed by low-pass filtering.

After the driving signal and the additional $90^{\circ}$ phase shift, the demodulation switch control signal is generated by the comparator.
The front-end detection circuit adopts the charge sensitive amplifier to realize the continuous time voltage detection and completes the $\mathrm{C} / \mathrm{V}$ conversion. The circuit diagram is shown in Figure 2.

The feedback resistor is made up of a $\mathrm{T}$ resistor network that is biased in the linear region of the POMS tube and the small resistor. The equivalent feedback resistance is Req, and the operational amplifier is a low noise amplifier. The transfer function of the capacitance variable $\Delta \mathrm{Cs}$ to the output voltage Vout $=$ Vop-Von is expressed as follows:

$$
\mathrm{H}(\mathrm{s})=\frac{\text { Vout }}{\Delta C_{s}}=\frac{\left(V_{M I D}-V_{C M I N}\right) R_{e q} s}{1+R_{e q} C_{f} s}
$$

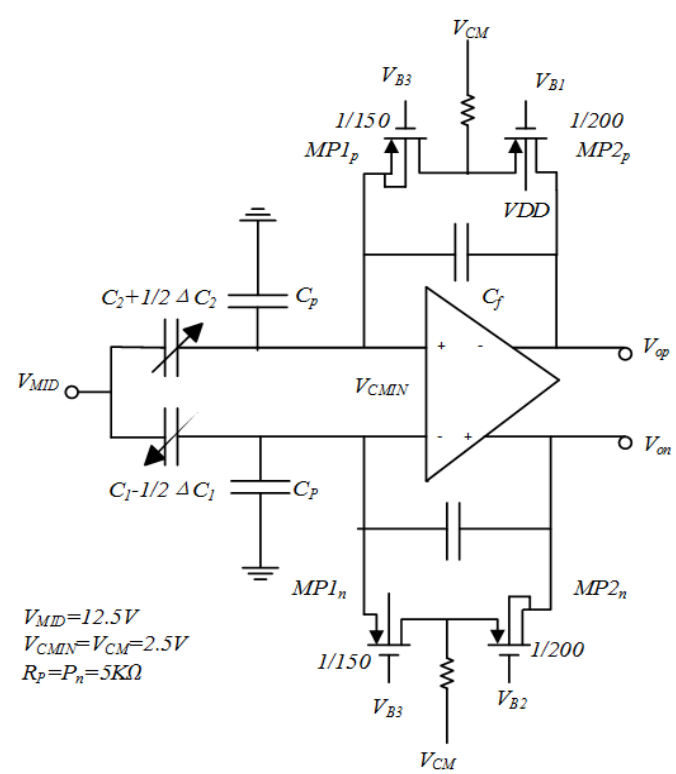

Figure 2: Front end $C / V$ conversion circuit

According to the formula (1), in order to stabilize the front-end $\mathrm{C} / \mathrm{V}$ conversion circuit, the voltage at both ends of the detection capacitor must be maintained constant. The op amp can stabilize the input voltage VCMINto $2.5 \mathrm{~V}$ through common mode feedback. The bias voltage on the intermediate plate is V MID $=12.5$ $\mathrm{V}$. The feedback capacitance is $5 \mathrm{pF}$. When the equivalent feedback resistance Reqis large enough, a conversion gain of $2.0 \times 1012 \mathrm{~V} / \mathrm{F}$ can be obtained.

The low pass filter is used to attenuate the high frequency signal produced during demodulation. After the coupling switch, the output signal contains $8.4 \mathrm{kHz}$ and $16.8 \mathrm{kHz}$ higher harmonic components. However, the angular rate signal frequency band is $0-100 \mathrm{~Hz}$. Although the second order analog lowpass filter can attenuate the harmonic to a very low level, the time constant of the second order filter achieves a cutoff frequency of $100 \mathrm{~Hz}$.

This requires a great deal of resistance and capacitance. In integrated circuits, large capacitive resistors occupy a large area, and the deviation of resistance is very large.

Therefore, it is difficult to set the cut-off frequency very accurate. In addition, the secondorder low-pass filter on the band of the signal attenuation is also great. Since the switchedcapacitor filter can be used to switch resistors and capacitances, and the cutoff frequency depends on the capacitance ratio and the switching frequency, the cutoff frequency can be made very accurate. Therefore, the switched-capacitor filter is used. However, the switching constant volume filter is a discrete time sampling system. In order to avoid spectral aliasing, anti-aliasing filters can be added to the switched-capacitor filter, and the output of the switched-capacitor filter contains many clockinduced glitches and spikes. Therefore, it is necessary to add a filter after the switched capacitor filter to filter out the high frequency components caused by the clock. 


\section{Result Analysis and Discussion}

The following is an overall simulation of the readout circuit. When the input angular rate is $\mathrm{DC}$, the capacitance change is the amplitude of the constant sine wave. When the sinusoidal angular rate is input, the capacitance change is an amplitude modulated wave whose envelope is sinusoidal.

According to the above changes, the Verilog-A language was used to test the differential capacitance model, and Cadence simulation software Specter was used to simulate the circuit.

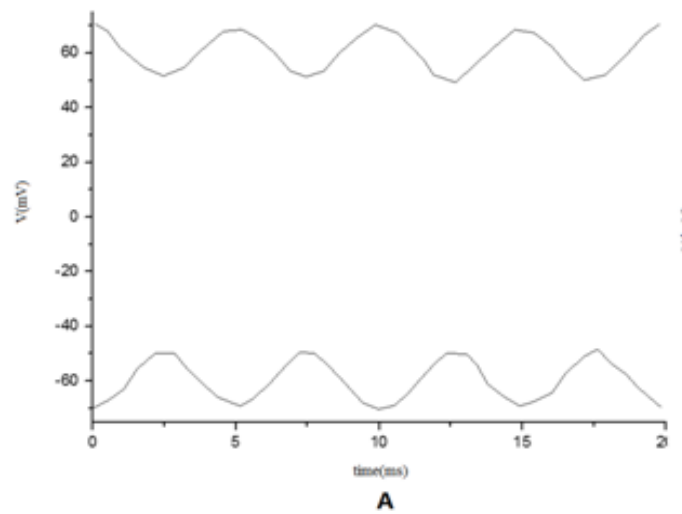

After the simulation, the input is a DC angular rate signal and contains a quadrature error, and the circuit can separate the angular rate signal from the quadrature.

In actual test, besides measuring direct-current (DC) input, alternating current (AC) input should be measured. The input angular rate is $100 \mathrm{~Hz}$, and the capacitance variation range is $50 \mathrm{fF}$. It contains the quadrature error input and output. It can be seen that the circuit can correctly demodulate the angular rate signal. As a result, the error correction method is corrected as shown in Figure 3.

Figure 3: Capacitance change and angular rate signal output (A: Capacitance change signal B: Angular rate signal)

Table 2. The circuit output at different frequencies and amplitudes

\begin{tabular}{|c|c|c|c|c|c|}
\hline Amplitude & $5 f F$ & $10 f F$ & $25 f F$ & $50 f F$ & $100 f F$ \\
\hline$D C$ & $36.2 \mathrm{mV}$ & $67.9 \mathrm{mV}$ & $163.9 \mathrm{mV}$ & $318.61 \mathrm{mV}$ & $639.2 \mathrm{mV}$ \\
\hline $5 \mathrm{~Hz}$ & $35.7 \mathrm{mV}$ & $67.6 \mathrm{mV}$ & $162.9 \mathrm{mV}$ & $318.45 \mathrm{mV}$ & $638.7 \mathrm{mV}$ \\
\hline $10 \mathrm{~Hz}$ & $35.5 \mathrm{mV}$ & $67.3 \mathrm{mV}$ & $162.5 \mathrm{mV}$ & $318.42 \mathrm{mV}$ & $638.4 \mathrm{mV}$ \\
\hline $25 \mathrm{~Hz}$ & $35.3 \mathrm{mV}$ & $65.7 \mathrm{mV}$ & $162.3 \mathrm{mV}$ & $318.36 \mathrm{mV}$ & $637.7 \mathrm{mV}$ \\
\hline $50 \mathrm{~Hz}$ & $34.8 \mathrm{mV}$ & $65.1 \mathrm{mV}$ & $160.6 \mathrm{mV}$ & $318.29 \mathrm{mV}$ & $637.3 \mathrm{mV}$ \\
\hline $100 \mathrm{~Hz}$ & $34.3 \mathrm{mV}$ & $64.1 \mathrm{mV}$ & $159.7 \mathrm{mV}$ & $318.23 \mathrm{mV}$ & $635.8 \mathrm{mV}$ \\
\hline
\end{tabular}

Table 2 is the circuit output at different frequencies and amplitudes. The amount of capacitance change is $50 \mathrm{fF}$, and the amount of quadrature error capacitance is 50fF. After error correction algorithm, compared with the absence of correction, the error rate dropped to about $0.3 \%$.It can be seen that the algorithm can effectively reduce the error caused by demodulation.

After calibration, the average capacitance of the circuit capacitance is $6.36 \mathrm{mV} / \mathrm{fF}$.

Table 3. The simulation noise of the readout circuit at different temperatures and process angles

\begin{tabular}{|c|c|c|c|c|c|}
\hline Frequency & $5 \mathrm{~Hz}$ & $10 \mathrm{~Hz}$ & $25 \mathrm{~Hz}$ & $50 \mathrm{~Hz}$ & $100 \mathrm{~Hz}$ \\
\hline $\begin{array}{c}\text { Output before } \\
\text { correction }\end{array}$ & $360.99 \mathrm{mV}$ & $359.41 \mathrm{mV}$ & $357.19 \mathrm{mV}$ & $355.92 \mathrm{mV}$ & $355.6 \mathrm{mV}$ \\
\hline $\begin{array}{c}\text { Error before } \\
\text { correction }\end{array}$ & $13.7 \%$ & $13.2 \%$ & $12.5 \%$ & $12.1 \%$ & $12 \%$ \\
\hline $\begin{array}{c}\text { Output after } \\
\text { correction }\end{array}$ & $318.45 \mathrm{mV}$ & $318.42 \mathrm{mV}$ & $318.36 \mathrm{mV}$ & $318.29 \mathrm{mV}$ & $318.23 \mathrm{mV}$ \\
\hline $\begin{array}{c}\text { Error after } \\
\text { correction }\end{array}$ & $0.3 \%$ & $0.29 \%$ & $0.27 \%$ & $0.25 \%$ & $0.23 \%$ \\
\hline
\end{tabular}


Table 3 is the simulation noise of the readout circuit at different temperatures and process angles. As the temperature changes, many parameters of resistors, transistors and other components will change, which will lead to an equivalent feedback resistance. The noise of the circuit, such as operational amplifier and high pass filter, has a certain influence on the noise of the readout circuit. Generally speaking, the noise of the circuit is worse because of the increase of temperature.

The readout circuit designed in this paper is implemented by $5 \mathrm{~V}$ and $0.25 \mu \mathrm{m}$ process. When the process angle is TT and the temperature is 27 degrees, the noise is $0.776 \mathrm{aF} / \sqrt{\mathrm{Hz}}$ and the bandwidth is $100 \mathrm{~Hz}$. The noise within the signal bandwidth can be detected with a capacitance change of

$$
\begin{aligned}
& C_{s_{2} \text { noise }}=\frac{0.076 \mathrm{aF}}{\sqrt{\mathrm{Hz}}} \times \sqrt{100 \mathrm{~Hz}}=7.76 \mathrm{aF} \\
& \text { When the maximum capacitance is } \\
& C_{s_{2} \text { noise }}=100 \mathrm{fF} \text { the signal-to-noise ratio is } \\
& \text { calculated to be } 82.2 \mathrm{~dB} .
\end{aligned}
$$

\section{Conclusion}

The design process of micromachined gyroscope readout circuit is mainly studied. Firstly, the parameters and electrical model of micromechanical gyroscope are introduced. Secondly, the design of the readout circuit is given, and then the design of each module in the program is described in detail. It includes noise tuning of front-end $\mathrm{C} / \mathrm{V}$ conversion circuit, $\mathrm{T}$ shape feedback resistor network, high pass filter, coupled switch, phase-shift circuit and lowpass filter. Its noise and transmission characteristics are analyzed and simulated. Finally, the whole readout circuit is simulated.

Under the BiCOMS $0.25 \mu \mathrm{m}$ process, the design of readout circuit for capacitive micromachined gyroscope with high sensitivity and low noise has been realized. When the temperature is $300 \mathrm{~K}$ and the process angle is TT, the sensitivity is $6.36 \mathrm{mV} / \mathrm{fF}$, and the noise is $0.776 \mathrm{aF} / \mathrm{Hz}$. When the maximum capacitance is $100 \mathrm{fF}$ and the bandwidth is $100 \mathrm{~Hz}$, the signal-to-noise ratio is $82.2 \mathrm{~dB}$.

Due to limited time, the entire circuit layout has not been completed. The next step is to complete the layout work. After the completion of the circuit layout design, the simulation experiment is carried out to observe whether the influence of parasitic and mismatch on the circuit can be ignored, and how to deal with these problems. Test the resulting circuit by streaming, which includes parameters such as noise, sensitivity, and so on. Thus, the actual performance of the chip is obtained.

\section{References}

[1] Rombach S, Marx M, Nessler S, et al. An Interface ASIC for MEMS Vibratory Gyroscopes with a Power of $1.6 \mathrm{~mW}, 92 \mathrm{~dB}$ DR and $0.007^{\circ} / \mathrm{s} / \$ \backslash \mathrm{sqrt}$ $\{\backslash \mathrm{rm}\{\mathrm{Hz}\}\}$ \$ Noise Floor Over a $40 \mathrm{~Hz}$ Band [J]. IEEE Journal of Solid-State Circuits, 2016, 51(8): 1915-1927. https://doi.org/10.1109/jssc.2016.2571670

[2] Do C, Erbes A, Yan J, et al. Design and implementation of a low-power hybrid capacitive MEMS oscillator [J]. Microelectronics Journal, 2016, 56: 1-9. https://doi.org/10.1016/j.mejo.2016.07.007

[3] Laghi G, Marra C R, Minotti P, et al. A 3-D micromechanical multi-loop magnetometer driven off-resonance by an on-chip resonator [J]. Journal of Microelectromechanical Systems, 2016, 25(4): 637-651. https://doi.org/10.1109/jmems.2016.2563180

[4] Aezinia F, Bahreyni B. Low-power, parasiticinsensitive interface circuit for capacitive microsensors [J]. IET Circuits, Devices \& Systems, 2016, 10(2): 104-110. https://doi.org/10.1049/iet-cds.2015.0077

[5] Baranov P, Nesterenko T, Tsimbalist E, et al. The stabilization system of primary oscillation for micromechanical gyroscope [J]. Measurement Science and Technology, 2017, 28(6). https://doi.org/10.1088/1361-6501/aa66c6

[6] Busurin V I, Pham A T. Micro-opto-electromechanical angular velocity transducer based on the optical tunneling effect [J]. Optoelectronics, Instrumentation and Data Processing, 2016, 52(2): 210-215. https://doi.org/10.3103/s8756699016020151

[7] Gholamzadeh R, Jafari K, Gharooni M. Design, simulation and fabrication of a MEMS accelerometer by using sequential and pulsedmode DRIE processes [J]. Journal of Micromechanics and Microengineering, 2016, 27(1): 015022. https://doi.org/10.1088/1361$6439 / 27 / 1 / 015022$ 